\title{
Urinary Tract infection, Drug Resistance Profile and Fetal Outcomes among Pregnant women in Selected Health Facilites of Addis Ababa, Ethiopia: A cross-sectional study design
}

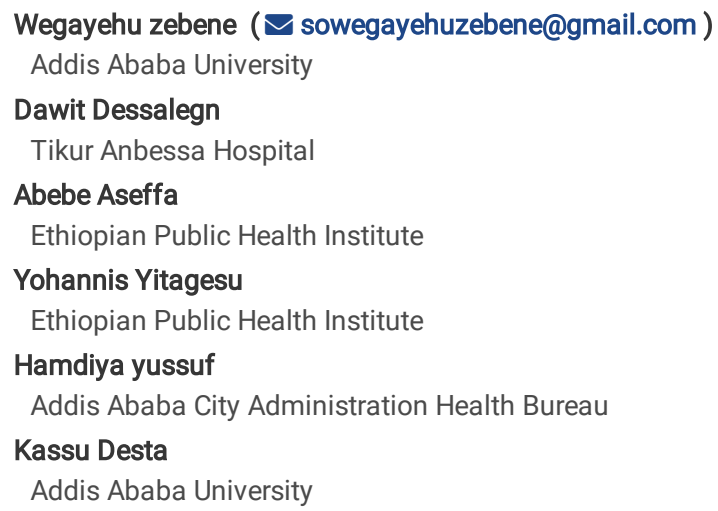




\section{Abstract}

Background: Urinary tract infection (UTI) is a commonly encountered health problem among pregnant women. Untreated UTI may result in fetal complications like preterm birth, low birth weight, intra uterine growth retardation and intrauterine fetal death.

One of the major problems with bacterial pathogens is their ability to develop drug resistance. Determining drug susceptibility pattern of bacteria from urinary tract helps to identify effective drugs and minimize further adverse perinatal outcomes. The purpose of this study was to describe the magnitude and drug resistance profile of pregnant women and related fetal outcomes.

Methods: A cross-sectional study was conducted from March to June 2019. Socio-demographic data of the study participants was collected by administering structured questionnaire after obtaining full consent of the participants. Clean catch mid-stream urine was collected from the study participants and the samples were transported to the laboratory with screw-capped container. Blood and MacConkey agar were used to cultivate bacteria from urine sample. Bacterial colonies were isolated and identified using standard biochemical properties. Antibacterial susceptibility test was done on Muller-Hinton agar using antibiotic discs. At the time of delivery, infant's birth weight and gestational age was recorded on the log book as part of the daily work. Fetal outcome data were recorded from the log book and entered to EPI data management software then transferred to SPSS for analysis.

Results: Out of 424 urine samples processed, 63 (14.9\%) yielded significant bacteriuria. Fifty-nine out of 63 (93.7\%) cases of UTIs were recorded among age group of 15-34 years. Escherichia coli, Klebsiella pneumoniae, and Staphylococcus aureus were the three dominant bacterial isolates.

Most of the gram negative bacterial isolates were resistant to ampicillin and ceftazidime (43.2\% each) while gram positive was resistant for tetracycline $(36.8 \%)$. The result of this study showed that UTI had statistical association with the occurrence of Intrauterine Fetal Death (IUFD) and Prerupture of Membrane (PROM) $(\mathrm{P}<.05)$.

Conclusion: Adverse fetal outcomes are significantly linked with the presence of UTI during pregnancy. The condition can be managed if early diagnosis and treatments are made for the mother.

\section{Background}

The urinary tract includes the organs that collect and store urine and release it from the body and these organs include the kidneys, ureters and bladder, urethra and accessory structures [1]. A urinary tract infection (UTI) is an infection caused by growth of microbes to different structures involved in formation and elimination of urine namely the kidney, ureters, urinary bladder and urethra [2]. Based on the structures involved, UTIs are medically classified as cystitis (if the infection is in the bladder), pyelonephritis (if kidney is affected by the infection), ureteritis (ureter infection) and urethritis (infection in the urethra) [2].

UTI affects both genders but it is more prevalent (75\%) in females [3], due to short urethra and contamination of the urinary tract with fecal flora from the anal opening $[4,5]$. In non-pregnant female, the uterus lies behind and partly over the bladder [6]. During the course of pregnancy, the enlarging uterus creates a pressure on nearby ureters and bladder which results a delay in urine flow and prevent complete emptying of urine; this condition places an opportunity for growth of microbes within the urinary tract [7]. In addition to the static urine in dilated urethra, development of glycosuria further enhances bacterial growth in the urethra and ascending tract. Furthermore; an increased bladder volume along with decreased bladder and urethral tone enhances bacterial growth in pregnant women $[6,8,9]$.

UTI is a common health problem for almost $50 \%$ of women during their child bearing age (16-35) and second most common illness after anemia for more than $10 \%$ of pregnant women $[2,10,11]$. UTI-related adverse fetal outcomes in untreated case include; low birth weight, intrauterine growth retardation (IUGR), intrauterine fetal death and preterm delivery $[5,10,12]$.

Escherichia coli is responsible for the majority of UTI cases and Staphylococcus saprophyticus, Klebsiella species, Proteus species and Enterobacter species account for the remaining cases $[2,13,14]$.

Nitrofurantoin, amikacin, ofloxacin, ciprofloxacin and cefotaxim were highly effective drugs over most bacterial isolates in Libya, while previous study in Ethiopia by Alemu, A., et al., reported similar finding for ceftriaxon, ciprofloxacin, norfloxacin, gentamicin, amoxicillin-clavulanic acid, co-trimoxazole. In this study, chloramphenicol produced the highest sensitivity to all isolates $[6,15]$. According to the report from similar study conducted in Dire Dawa, Eastern Ethiopia, gram negative bacteria were highly sensitive for ceftriaxone, gentamicin, and ciprofloxacin [12].

All the isolates were resistant to nalidixic acid, cotrimoxasole and cefaloxine in Nigeria, [16]

E.coli was highly sensitive to nitrofurantoin, cefotaxim, amikacin, ciprofloxacin and ofloxacin in Nigeria [15] to ceftriaxone, gentamicin, ciprofloxacin, trimethoprim-sulfamethoxazole, and chloramphenicol in Dire Dawa, Ethiopia [12], to ceftriaxon, chloramphenicol, ciprofloxacin, norfloxacin and gentamicin in Gondar, Ethiopia [6], and to nitrofurantoin in Bahir Dar, Ethiopia [17].

The finding of the study conducted by Derese et.al in Dire Dawa, Eastern Ethiopia indicates that gram-positive isolates were susceptible to gentamicin, erythromycin, ceftriaxone, ciprofloxacin and nitrofurantoin. According to this study, the isolates were resistant to ampicillin, tetracycline, and trimethoprimsulfamethoxazole [12]. Similar study conducted in Bahir Dar, Northern Ethiopia reported the high sensitivity of gram positive bacterial isolates to gentamicin, nitrofurantoin and amoxicillin-clavulnic acid [17].

In cross sectional retrospective study conducted in Iran, UTI during pregnancy and fetal weight were highly associated [18]. Similarly, a study in Ghana revealed that birth weight, birth outcome (live birth or still birth), APGAR score at 1 minute and gestational age are significantly dependent on the UTI status of 
the mother during pregnancy [19]. Iqbal et. al. also reported that, $11.3 \%$ of the deliveries from UTI positive pregnant mothers produced low birth weighted babies [5].

If they are left undetected and untreated, they may cause adverse maternal and fetal outcomes [20]. To this end determining uropathogen isolates and their drug susceptibility pattern among pregnant women is of a highest priority before deciding to manage UTI cases [21]. Two factors- Poor patient compliance and incomplete course of antibiotic therapy are responsible for the evolution of resistant bacterial strains to most of antibiotics [3, 22].

The distribution of antimicrobial susceptibility data of UTI causing microorganisms changes from time to time and from place to place [23]. This urges for the isolation of bacterial strains and their drug resistance profile for that specific location before treatment is given based on sign and symptoms of patients.

There should be periodic monitoring of etiologic agents of UTI and their resistance pattern to avoid further complication of the case [24]. Therefore, screening for UTI in pregnant women is very important if the prevalence rates are $\geq 2 \%$ [25]

The studies regarding uropathogens and their antimicrobial susceptibility patterns in pregnant women and the related fetal complications in Ethiopia are limited [26].

Studies have shown the strong association that exist between maternal UTI during pregnancy and fetal complications including low birth weight, APGAR score, PROM, and IUFD $[5,18,19]$.

Thus; the result of this study provide vital information on type of bacterial isolates and the drug resistance pattern in relation to fetal complications in pregnant women attending at study sites.

\section{Methods}

All pregnant women on their third trimester during the study period and attending antenatal clinic at the two health centers, and Tikur Anbessa Speclised Hospital that fulfil the inclusion criteria were the study population.

Four hundred twenty-four (424) participants were involved in the study by using single proportion formula.

Every pregnant mothers attending the antenatal clinic who were willing and available at the time of study, fulfilling the inclusion and exclusion criteria were enrolled by non-probable consecutive technique until the number reaches the required sample size.

A structured questionnaire was used to collect socio-demographic data and clinical history of the study participants. Age, marital status, educational status, UTI sign and symptoms and questions related to fetal outcomes were included on the questionnaire. Participant's clinical data and fetal outcome data were taken from the individual card and ANC log books.

Midstream urine sample were collected from the study participants from March to June 2019. Participants were informed on how to bring "mid-stream" urine of 15-20 $\mathrm{ml}$ volume through the following process.

QThorough hand washes with water and dry

$\triangle$ Wash the genitalia

$\triangle$ Void the first $20-30 \mathrm{ml}$ of urine in the toilet then collect the remaining urine in to a sterile clean container.

Then, the samples were transported to Ethiopian Public Health Institute (EPHI) microbiology laboratory within an hour after it was collected. All urine specimens were collected in a well labeled, sterile, dry, wide-necked, leak proof and screw capped container.

A calibrated sterile wire and plastic disposable loops were used to inoculate $1 \mu$ of each urine specimen on MacConkey and blood agar plates.

Identification of the isolated bacterial pathogens were done on the basis of culture morphology and biochemical characteristics. Indole test, citrate, urea, coagulase, catalase, motility agar, lysine iron agar and novobiosin disk were used for identification purpose.

Antimicrobial Susceptibility testing were performed on isolates according to the criteria of Clinical and Laboratory Standards Institute (CLSI, 2019) using the Kirby-Bauer disc diffusion method on Muller-Hinton Agar. A homogeneous bacterial suspension was prepared by mixing a loop full of pure bacterial colony in $5 \mathrm{ml}$ of $0.85 \%$ sterile saline solution. After adjusting the $0.5 \mathrm{McFarland}$ standard of the suspension, it was evenly inoculated on Muller Hinton agar plate using sterile cotton swab and antibiotic disks were placed at $15 \mathrm{~mm}$ and $24 \mathrm{~mm}$ distance from the edge and from each other respectively. The plates were then incubated for 24 hours at $37^{\circ} \mathrm{C}$. The isolates were categorized as sensitive (S), resistant (R) or intermediate (I) based on the inhibition zone they produced around the antibiotics used following the described break point in CLSI.

Confirmation of AST result was done by Vitek 2 Compact system. AST- GN73 and AST-GP74 cards were used for the susceptibility testing of gram negative and gram positive bacteria respectively.

The drugs that were used in the susceptibility test of gram negative bacteria are Ampicillin $(10 \mu \mathrm{g})$, Ampicillin/Sublactam (10 $\mu \mathrm{g})$, Piperacillin/ Tazobactam $(10 \mu \mathrm{g})$, Cefazolin $(30 \mu \mathrm{g})$, Cefoxitin $(30 \mu \mathrm{g})$, Ceftazidime $(30 \mu \mathrm{g})$, Ceftriaxone $(30 \mu \mathrm{g})$, Cefepime $(30 \mu \mathrm{g})$, Meropenem (10 $\mu \mathrm{g})$, Amikacin $(10 \mu \mathrm{g})$, Gentamicin $(10 \mu \mathrm{g})$, Tobramycin $(10 \mu \mathrm{g})$, Ciprofloxacin $(5 \mu \mathrm{g})$, Levofloxacin $(5 \mu \mathrm{g})$, Nitrofurantoin $(300 \mu \mathrm{g})$, Trimethoprim/ Sulfamethoxazole $(1.25 \mu \mathrm{g})$. While for gram positive bacteria, Amoxicillin $(10 \mu \mathrm{g})$, Penicillin $(5 \mu \mathrm{g})$, Cefotaxime $(30 \mu \mathrm{g})$, Ceftriaxone $(30 \mu \mathrm{g})$, Chloramphenicol $(30 \mu \mathrm{g})$, Ertapenem $(10 \mu \mathrm{g})$, Erythromycin 
$(15 \mu \mathrm{g})$, Levofloxacin $(5 \mu \mathrm{g})$, Linezolid $(30 \mu \mathrm{g})$, Meropenem $(10 \mu \mathrm{g})$, Moxifloxacin $(5 \mu \mathrm{g})$, Ofloxacin $(5 \mu \mathrm{g})$, Telithromycin, Tetracycline $(30 \mu \mathrm{g})$, Trimethoprim/Sulfamethoxazole $(1.25 \mu \mathrm{g})$, Vancomycin were used.

During data collection and sample collection, data quality was ensured through the use of standardized questionnaire, proper orientation and intensive supervision by the principal investigator. In addition, the questionnaires are translated in to Amharic language for easy understanding. SOPs of the microbiology laboratory were followed.

Sterility of the media was checked by overnight incubation of the media at $37^{0} \mathrm{c}$ prior to inoculation. Quality control bacteria were used to check the growth support of the media. For blood agar plate (S. pyogen, E. faecalis, S pneumonia), for Mackonkey agar plate (E. coli, P. mirabilis, E. faecalis) and for Muller Hinton agar plate (S. aureus, E. coli, E. faecalis) were used. All the data were checked for their completeness and representativeness prior to entry.

Data entry was done using EpiData Manager (version 4.4.2.1) and statistical analysis was performed using IBM SPSS (version 23) software.

Findings of the study was further explained in words and tables. Binary and multiple logistic regression analysis was used to see if there is an association with different variables. In all cases P-value less than 0.05 considered as statistically significant.

\section{Results}

\subsection{Socio demographic characteristics}

There were a total of 424 participants and the age distribution of study subjects ranges from 18-40 years with the mean (standard deviation) of $27 \pm 4.6$ years and $393(92.7 \%)$ of them belongs to the age group 15-34 years.

It was observed that around $1.4 \%$ of the study participants were divorced. Only 41 (9.7\%) of the study participants had higher education and 293 (69.1\%) were housewives. Based on their gravidity, more than half of the study participants (62.3\%) were multigravida and 80 (18.9\%) of the study participants had history of previous urinary tract infection. None of the study subjects were cigarette or alcohol addicted (Table 1).

Table 1: Sociodemographic, obstetric, and clinical variables of pregnant women, March-June, 2019. 


\begin{tabular}{|c|c|c|c|c|c|}
\hline \multirow[t]{2}{*}{ Variables } & \multicolumn{5}{|c|}{ Total No of participants $=424$} \\
\hline & № (\%) & No Bacteriuria № (\%) & $\begin{array}{l}\text { Bacteriuria } \\
\text { № (\%) }\end{array}$ & $\mathrm{Chi}^{2}$ & $P$ value \\
\hline Age & $138(32.4)$ & $118(27.8)$ & $20(4.7)$ & 0.147 & 0.929 \\
\hline $15-24$ & $255(60.3)$ & $216(50.9)$ & $39(9.2)$ & & \\
\hline $25-34$ & $31(7.3)$ & $27(6.4)$ & $4(0.9)$ & & \\
\hline \multicolumn{6}{|l|}{$35-44$} \\
\hline Marital Status & $417(98.6)$ & $356(84.2)$ & $61(14.4)$ & 1.633 & 0.201 \\
\hline Married & $6(1.4)$ & $4(0.9)$ & $2(0.5)$ & & \\
\hline \multicolumn{6}{|l|}{ Divorced } \\
\hline Educational level & $68(16.0)$ & $55(13)$ & $13(3.1)$ & 9.451 & 0.051 \\
\hline Illiterate & $173(40.8)$ & $147(34.7)$ & $26(6.1)$ & & \\
\hline Elementary & $142(33.5)$ & $127(30)$ & $15(3.5)$ & & \\
\hline High school & $41(9.7)$ & $32(7.6)$ & $9(2.2)$ & & \\
\hline \multicolumn{6}{|l|}{ Higher education } \\
\hline Occupation & $293(69.1)$ & $248(58.5)$ & 45 (10.6) & 0.622 & 0.891 \\
\hline Housewife & $78(18.4)$ & $67(15.8)$ & $11(2.6)$ & & \\
\hline Government & $29(6.8)$ & $26(6.1)$ & $3(0.7)$ & & \\
\hline Non-Government & $24(5.7)$ & $20(4.7)$ & $4(0.9)$ & & \\
\hline \multicolumn{6}{|l|}{ Student } \\
\hline Gravidity & $160(37.7)$ & $133(31.4)$ & $27(6.4)$ & 1.417 & 0.234 \\
\hline Primigravida & $264(62.3)$ & $228(53.8)$ & $36(8.5)$ & & \\
\hline \multicolumn{6}{|l|}{ Multigravida } \\
\hline History of UTI & $80(18.9)$ & $68(16)$ & $12(2.8)$ & 0.002 & 0.968 \\
\hline Yes & $344(81.1)$ & $293(69.1)$ & $51(12)$ & & \\
\hline \multicolumn{6}{|l|}{ No } \\
\hline Cigarette/ alcohol addiction & 0 & $0(0)$ & $0(0)$ & - & - \\
\hline Yes & $424(100)$ & $361(85.1)$ & $63(14.9)$ & & \\
\hline \multicolumn{6}{|l|}{ No } \\
\hline Symptoms of UTI & $82(19.3)$ & $68(16.0)$ & $14(3.3)$ & 4.915 & 0.555 \\
\hline Yes & $342(80.7)$ & $293(69.1)$ & 49 (11.6) & & \\
\hline No & & & & & \\
\hline
\end{tabular}

\subsection{Magnituide of UTI and distribution of bacterial isolates}

Four hundred twenty-four (424) urine samples were collected from pregnant women attending antenatal clinic at the study sites, out of which 63 (14.9\%) were positive for the presence of significant bacteriuria (Figure 1).

From the total study subjects, $80.7 \%$ of them had no symptoms of UTI out of which $11.6 \%$ had significant bacteriuria in their urine.

Of the total isolates, gram negative bacteria were the most common accounting for $44(68.75 \%)$ of the total isolates. E. coli, K. pneumonia and S. aureus were the three predominant bacteria consisting of $28(44.4 \%), 6(9.5 \%)$ and $6(9.5 \%)$ of the total isolates respectively. The least abundant bacterial isolates were $C$. freundii, K. oxytoca, and P. stuartii, each represented by one (1.6\%) isolates (Figure 2).

\subsection{Antimicrobial susceptibility pattern of isolated bacteria}

Tables 2 and 3 summarized the antibiotic susceptibility patterns of gram negative and positive isolates respectively. 


\begin{tabular}{|c|c|c|c|c|c|c|c|c|c|c|c|c|c|c|c|c|}
\hline \multirow{2}{*}{$\begin{array}{l}\text { Bacterial } \\
\text { Isolates }\end{array}$} & \multirow[t]{2}{*}{ № } & \multirow[t]{2}{*}{ Pattem } & \multicolumn{14}{|c|}{ Antimicrobial agent tested } \\
\hline & & & $\mathrm{AM}$ & SAM & $\mathrm{TM}$ & $\mathrm{CZ}$ & FOX & CAZ & CRO & FEP & MEM & AMK & GM & ТОВ & CIP & LEV \\
\hline \multirow{6}{*}{$\begin{array}{l}\text { A. } \\
\text { baumannii }\end{array}$} & \multirow[t]{6}{*}{2} & \multirow[t]{2}{*}{ S } & 1 & 1 & 2 & 2 & 2 & 1 & 2 & \multirow{2}{*}{$\begin{array}{l}2 \\
(100)\end{array}$} & 1 & \multirow{2}{*}{$\begin{array}{l}2 \\
(100)\end{array}$} & 2 & 2 & 2 & 2 \\
\hline & & & (50) & (50) & $(100)$ & $(100)$ & $(100)$ & (50) & $(100)$ & & (50) & & $(100)$ & (100) & $(100)$ & $(101$ \\
\hline & & \multirow[t]{2}{*}{$\mathrm{R}$} & 1 & 0 & 0 & 0 & 0 & 1 & 0 & 0 & 0 & 0 & 0 & 0 & 0 & 0 \\
\hline & & & (50) & (0) & (0) & $(0)$ & $(0)$ & (50) & $(0)$ & (0) & (0) & (0) & (0) & $(0)$ & (0) & (0) \\
\hline & & \multirow[t]{2}{*}{ । } & 0 & 1 & 0 & 0 & 0 & 0 & 0 & 0 & 1 & 0 & 0 & 0 & 0 & 0 \\
\hline & & & (0) & (50) & $(0)$ & (0) & (0) & (0) & $(0)$ & (0) & $(50)$ & (0) & (0) & (0) & $(0)$ & (0) \\
\hline \multirow{6}{*}{ diversus } & \multirow[t]{6}{*}{2} & \multirow[t]{2}{*}{ S } & 0 & 1 & 2 & 1 & 2 & 0 & 2 & \multirow{2}{*}{$\begin{array}{l}2 \\
(100)\end{array}$} & 2 & \multirow{2}{*}{2} & 2 & 2 & 2 & 2 \\
\hline & & & (0) & (50) & $(100)$ & (50) & (100) & (0) & $(100)$ & & $(100)$ & & $(100)$ & (100) & $(100)$ & $(101$ \\
\hline & & \multirow[t]{2}{*}{$\mathrm{R}$} & 1 & 0 & 0 & 1 & 0 & 1 & 0 & 0 & 0 & 0 & 0 & 0 & 0 & 0 \\
\hline & & & (50) & $(0)$ & (0) & (50) & (0) & (50) & (0) & (0) & (0) & $(0)$ & (0) & (0) & (0) & (0) \\
\hline & & \multirow[t]{2}{*}{ I } & 1 & 1 & 0 & 2 & 0 & 1 & 0 & 0 & 0 & 0 & 0 & 0 & 0 & 0 \\
\hline & & & (50) & (50) & (0) & (100) & (0) & (50) & (0) & (0) & (0) & $(0)$ & (0) & (0) & (0) & (0) \\
\hline \multirow[t]{6}{*}{ C. freundii } & \multirow[t]{6}{*}{1} & \multirow[t]{2}{*}{ S } & 0 & 0 & 1 & 0 & 1 & 0 & 0 & 0 & 0 & \multirow{2}{*}{$\begin{array}{l}1 \\
(100)\end{array}$} & 1 & 1 & 1 & 1 \\
\hline & & & (0) & $(0)$ & (100) & (0) & (100) & (0) & (0) & $(0)$ & (0) & & $(100)$ & (100) & (100) & $(101$ \\
\hline & & \multirow[t]{2}{*}{$\mathrm{R}$} & 1 & 1 & 0 & 1 & 0 & 1 & 1 & \multirow{2}{*}{$\begin{array}{l}1 \\
(100)\end{array}$} & 1 & 0 & 0 & 0 & 0 & 0 \\
\hline & & & $(100)$ & (100) & (0) & (100) & (0) & (100) & (100) & & (100) & (0) & (0) & (0) & (0) & (0) \\
\hline & & \multirow[t]{2}{*}{ I } & 0 & 0 & 0 & 0 & 0 & 0 & 0 & 0 & 0 & 0 & 0 & 0 & 0 & 0 \\
\hline & & & (0) & $(0)$ & (0) & (0) & (0) & $(0)$ & $(0)$ & $(0)$ & (0) & (0) & (0) & (0) & (0) & (0) \\
\hline \multirow[t]{8}{*}{ E. coli } & \multirow[t]{8}{*}{28} & $S$ & 11 & 18 & 24 & 25 & 26 & 14 & 23 & 26 & 20 & 26 & 25 & 27 & 25 & 26 \\
\hline & & & & $(64.3)$ & & (89.3) & & (50) & & & & & & & & \\
\hline & & $\mathrm{R}$ & 16 & 7 & 4 & 2 & 2 & 11 & 5 & 2 & 2 & 2 & 3 & 1 & 3 & 2 \\
\hline & & & & (25) & & (7.1) & (7.1) & & & (7.1) & $(7.1)$ & (7.1) & & (3.6) & & (7.1 \\
\hline & & $\mathrm{R}$ & 2 & 2 & 0 & 1 & 0 & 4 & 2 & 3 & 3 & 1 & 0 & 0 & 0 & 0 \\
\hline & & & & & (0) & (16.7) & (0) & (66.7) & & (50) & (50) & & (0) & (0) & (0) & (0) \\
\hline & & 1 & 0 & 0 & 0 & 0 & 0 & 0 & 0 & 0 & 1 & 0 & 0 & 0 & 0 & 0 \\
\hline & & & (0) & (0) & (0) & (0) & (0) & (0) & (0) & (0) & & (0) & (0) & (0) & (0) & (0) \\
\hline
\end{tabular}

Table 2: Antibiotic susceptibility pattern of Gram negative isolates, in selected health facilities of Addis Ababa, Ethiopia, March-June, 2019.

$\mathrm{AM}=$ Ampicillin, $\mathrm{SAM}=$ Ampicillin/Sublactam, $\mathrm{TM}=$ Piperacillin/ Tazobactam, CZ=Cefazolin, FOX=Cefoxitin, CAZ=Ceftazidime, CRO=Ceftriaxone, FEP= Cepepime, MEM= Meropenem, AMK= Amikacin, GM=Gentamicin, TOB=Tobramycin, $\mathrm{CIP}=$ Ciprofloxacin, LEV= Levofloxacin, FT=Nitrofurantoin, $S X T=$ Trimethoprim/ Sulfamethoxazole, $\mathrm{S}=$ Susceptible, $\mathrm{R}=$ Resistant, I=Intermediate 


\begin{tabular}{|c|c|c|c|c|c|c|c|c|c|c|c|c|c|c|c|c|}
\hline \multirow{2}{*}{$\begin{array}{l}\text { Bacterial } \\
\text { Isolates }\end{array}$} & \multirow[t]{2}{*}{ № } & \multirow[t]{2}{*}{ Pattern } & \multicolumn{14}{|c|}{ Antimicrobial agent tested } \\
\hline & & & AM & SAM & TM & $\mathrm{CZ}$ & FOX & CAZ & CRO & FEP & MEM & AMK & GM & ТОВ & CIP & LE \\
\hline \multirow[t]{6}{*}{ K.oxytoca } & \multirow[t]{6}{*}{1} & \multirow[t]{2}{*}{ S } & 0 & \multirow{2}{*}{$\begin{array}{l}1 \\
(100)\end{array}$} & 1 & 1 & 1 & 0 & 0 & \multirow{2}{*}{$\begin{array}{l}1 \\
(100)\end{array}$} & 0 & \multirow{2}{*}{$\begin{array}{l}1 \\
(100)\end{array}$} & 1 & 1 & 1 & 1 \\
\hline & & & (0) & & $(100)$ & (100) & $(100)$ & $(0)$ & (0) & & $(0)$ & & $(100)$ & $(100)$ & $(100)$ & $(10$ \\
\hline & & \multirow[t]{2}{*}{$\mathrm{R}$} & 1 & 0 & 0 & 0 & 0 & 1 & 1 & 0 & 1 & 0 & 0 & 0 & 0 & 0 \\
\hline & & & $(100)$ & $(0)$ & $(0)$ & (0) & $(0)$ & $(100)$ & $(100)$ & $(0)$ & $(100)$ & $(0)$ & $(0)$ & $(0)$ & $(0)$ & (0) \\
\hline & & \multirow[t]{2}{*}{1} & 0 & 0 & 0 & 0 & 0 & 0 & 0 & 0 & 0 & 0 & 0 & 0 & 0 & 0 \\
\hline & & & (0) & (0) & (0) & (0) & (0) & $(0)$ & $(0)$ & (0) & (0) & (0) & $(0)$ & (0) & (0) & (0) \\
\hline \multirow[t]{6}{*}{ P. stuartii } & \multirow[t]{6}{*}{1} & \multirow[t]{2}{*}{$\mathrm{s}$} & 1 & 0 & 0 & 1 & 1 & 1 & 1 & \multirow{2}{*}{$\begin{array}{l}1 \\
(100)\end{array}$} & 1 & \multirow{2}{*}{$\begin{array}{l}1 \\
(100)\end{array}$} & 1 & 1 & 1 & 1 \\
\hline & & & $(100)$ & (0) & (0) & (100) & $(100)$ & $(100)$ & $(100)$ & & (100) & & (100) & (100) & (100) & $(10$ \\
\hline & & \multirow[t]{2}{*}{$\mathrm{R}$} & 0 & \multirow{2}{*}{$\begin{array}{l}1 \\
(100)\end{array}$} & 1 & 0 & 0 & 0 & 0 & 0 & 0 & 0 & 0 & 0 & 0 & 0 \\
\hline & & & (0) & & $(100)$ & (0) & (0) & (0) & (0) & (0) & (0) & (0) & (0) & (0) & (0) & (0) \\
\hline & & \multirow[t]{2}{*}{1} & 0 & 0 & 0 & 0 & 0 & 0 & 0 & 0 & 0 & 0 & 0 & 0 & 0 & 0 \\
\hline & & & (0) & (0) & (0) & (0) & (0) & (0) & (0) & (0) & (0) & (0) & (0) & (0) & (0) & (0) \\
\hline \multirow{6}{*}{$\begin{array}{l}\text { P. } \\
\text { aeruginosa }\end{array}$} & \multirow[t]{6}{*}{3} & \multirow[t]{2}{*}{ S } & \multirow{2}{*}{$\begin{array}{l}2 \\
(66.7)\end{array}$} & \multirow{2}{*}{$\begin{array}{l}3 \\
(100)\end{array}$} & 3 & 3 & 3 & 1 & \multirow{2}{*}{$\begin{array}{l}1 \\
(33.3)\end{array}$} & \multirow{2}{*}{$\begin{array}{l}3 \\
(100)\end{array}$} & \multirow{2}{*}{$\begin{array}{l}2 \\
(66.7)\end{array}$} & 2 & 3 & 2 & 2 & 3 \\
\hline & & & & & $(100)$ & (100) & $(100)$ & (33.3) & & & & & (100) & & & (10 \\
\hline & & $\mathrm{R}$ & 1 & 0 & 0 & 0 & 0 & 2 & 2 & 0 & 1 & 1 & 0 & 1 & 1 & 0 \\
\hline & & & & (0) & (0) & (0) & (0) & (66.7) & & (0) & & & (0) & & & (0) \\
\hline & & I & 0 & 0 & 0 & 0 & 0 & 0 & 0 & 0 & 0 & 0 & 0 & 0 & 0 & 0 \\
\hline & & & (0) & (0) & (0) & (0) & (0) & (0) & (0) & (0) & (0) & $(0)$ & (0) & (0) & (0) & (0) \\
\hline Total № of & 44 & S & 19 & 28 & 39 & 38 & 42 & 19 & 33 & 38 & 28 & 40 & 41 & 42 & 40 & 42 \\
\hline & & & & & & (86.4) & & & (75) & & & & & & & \\
\hline & & $\mathrm{R}$ & 23 & 11 & 5 & 5 & 2 & 21 & 11 & 6 & 8 & 4 & 3 & 2 & 4 & 2 \\
\hline & & & & & & (11.4) & $(4.5)$ & & (25) & & & (9.1) & $(6.8)$ & (4.5) & $(9.1)$ & $(4.9$ \\
\hline & & 1 & 2 & 5 & 0 & 1 & 0 & 4 & 0 & 0 & 8 & 0 & 0 & 0 & 0 & 0 \\
\hline & & & (4.5) & & (0) & (2.3) & (0) & $(9.1)$ & (0) & (0) & & (0) & (0) & (0) & (0) & (0) \\
\hline
\end{tabular}

Table 2: Antibiotic susceptibility pattern of Gram negative isolate in selected health facilities of Addis Ababa, Ethiopia, March-June, 2019.

$\mathrm{AM}=$ Ampicillin, $\mathrm{SAM}=$ Ampicillin/Sublactam, $\mathrm{TM}=$ Piperacillin/ Tazobactam, CZ=Cefazolin, FOX=Cefoxitin, CAZ=Ceftazidime, CRO= Ceftriaxone,FEP=Cepepime,MEM=Meropenem, AMK=Amikacin, GM=Gentamicin, TOB=Tobramycin, CIP=Ciprofloxacin, LEV= Levofloxacin, FT=Nitrofurantoin, SXT=Trimethoprim/ Sulfamethoxazole, $\mathrm{S}=$ Susceptible, R=Resistant, I=Intermediat 


\begin{tabular}{|c|c|c|c|c|c|c|c|c|c|c|c|c|c|c|c|c|}
\hline \multirow{2}{*}{$\begin{array}{l}\text { Bacterial } \\
\text { Isolates }\end{array}$} & \multirow{2}{*}{ № } & \multirow[t]{2}{*}{ Pattern } & \multicolumn{14}{|c|}{ Antimicrobial agent tested } \\
\hline & & & AMX & $\mathbf{P}$ & CTX & CRO & C & ETP & $E$ & LEV & LNZ & MEM & MXF & OFL & TEL & TE \\
\hline \multirow[t]{5}{*}{ E. faecalis } & \multirow[t]{5}{*}{4} & S & $\begin{array}{l}2 \\
(50)\end{array}$ & $\begin{array}{l}3 \\
(75)\end{array}$ & $\begin{array}{l}3 \\
(75)\end{array}$ & $\begin{array}{l}4 \\
(100)\end{array}$ & $\begin{array}{l}4 \\
(100)\end{array}$ & $\begin{array}{l}3 \\
(75)\end{array}$ & $\begin{array}{l}2 \\
(50)\end{array}$ & $\begin{array}{l}4 \\
(100)\end{array}$ & $\begin{array}{l}3 \\
(75)\end{array}$ & $\begin{array}{l}4 \\
(100)\end{array}$ & $\begin{array}{l}4 \\
(100)\end{array}$ & $\begin{array}{l}4 \\
(100)\end{array}$ & $\begin{array}{l}2 \\
(50)\end{array}$ & $\begin{array}{l}2 \\
(5 c\end{array}$ \\
\hline & & \multirow[t]{2}{*}{$\mathrm{R}$} & 2 & 1 & 1 & 0 & 0 & 1 & 2 & 0 & 1 & 0 & 0 & 0 & 1 & 2 \\
\hline & & & $(50)$ & $(25)$ & $(25)$ & $(0)$ & $(0)$ & $(25)$ & $(50)$ & $(0)$ & $(25)$ & (0) & $(0)$ & $(0)$ & $(25)$ & $(5 c$ \\
\hline & & \multirow[t]{2}{*}{ I } & 0 & 0 & 0 & 0 & 0 & 0 & 0 & 0 & 0 & 0 & 0 & 0 & 1 & 0 \\
\hline & & & $(0)$ & $(0)$ & $(0)$ & $(0)$ & $(0)$ & $(0)$ & (0) & (0) & (0) & (0) & $(0)$ & (0) & (25) & (0) \\
\hline \multirow[t]{5}{*}{$\begin{array}{l}S . \\
\text { agalactiae }\end{array}$} & \multirow[t]{5}{*}{3} & $S$ & $\begin{array}{l}1 \\
(33.3)\end{array}$ & $\begin{array}{l}3 \\
(100)\end{array}$ & $\begin{array}{l}2 \\
(66.7)\end{array}$ & $\begin{array}{l}2 \\
(66.7)\end{array}$ & $\begin{array}{l}1 \\
(33.3)\end{array}$ & $\begin{array}{l}2 \\
(66.7)\end{array}$ & $\begin{array}{l}3 \\
(100)\end{array}$ & $\begin{array}{l}3 \\
(100)\end{array}$ & $\begin{array}{l}3 \\
(100)\end{array}$ & $\begin{array}{l}3 \\
(100)\end{array}$ & $\begin{array}{l}3 \\
(100)\end{array}$ & $\begin{array}{l}3 \\
(100)\end{array}$ & $\begin{array}{l}1 \\
(33.3)\end{array}$ & $\begin{array}{l}0 \\
(0)\end{array}$ \\
\hline & & \multirow[t]{2}{*}{$\mathrm{R}$} & 1 & 0 & \multirow{2}{*}{$\begin{array}{l}1 \\
(33.3)\end{array}$} & 0 & 2 & 1 & 0 & 0 & 0 & 0 & 0 & 0 & 2 & 3 \\
\hline & & & (33.3) & (0) & & $(0)$ & $(66.7)$ & (33.3) & (0) & (0) & $(0)$ & (0) & $(0)$ & $(0)$ & $(66.7)$ & $(1 C$ \\
\hline & & I & 1 & 0 & 0 & 1 & 0 & 0 & 0 & 0 & 0 & 0 & 0 & 0 & 0 & 0 \\
\hline & & & (33.3) & $(0)$ & $(0)$ & (33.3) & (0) & (0) & (0) & (0) & $(0)$ & $(0)$ & $(0)$ & (0) & (0) & (0) \\
\hline $\begin{array}{l}S . \\
\text { epidermidis }\end{array}$ & 4 & $S$ & $\begin{array}{l}3 \\
(75)\end{array}$ & $\begin{array}{l}4 \\
(100)\end{array}$ & $\begin{array}{l}4 \\
(100)\end{array}$ & $\begin{array}{l}4 \\
(100)\end{array}$ & $\begin{array}{l}3 \\
(75)\end{array}$ & $\begin{array}{l}4 \\
(100)\end{array}$ & $\begin{array}{l}1 \\
(25)\end{array}$ & $\begin{array}{l}2 \\
(50)\end{array}$ & $\begin{array}{l}3 \\
(75)\end{array}$ & $\begin{array}{l}4 \\
(100)\end{array}$ & $\begin{array}{l}2 \\
(50)\end{array}$ & $\begin{array}{l}3 \\
(75)\end{array}$ & $\begin{array}{l}4 \\
(100)\end{array}$ & $\begin{array}{l}1 \\
(25\end{array}$ \\
\hline & & $\mathrm{R}$ & 1 & 0 & 0 & 0 & 1 & 0 & 3 & 2 & 1 & 0 & 1 & 1 & 0 & 3 \\
\hline & & & $(25)$ & (0) & (0) & (0) & (25) & (0) & (75) & (50) & (25) & (0) & (25) & (25) & (0) & (75 \\
\hline & & I & 0 & 0 & 0 & 0 & 0 & 0 & 0 & 0 & 0 & 0 & 1 & 0 & 0 & 0 \\
\hline & & & (0) & (0) & (0) & (0) & (0) & (0) & (0) & (0) & (0) & (0) & (25) & (0) & (0) & (0) \\
\hline S. aureus & 6 & $S$ & 6 & 5 & 5 & 4 & 4 & 4 & 5 & 4 & 6 & 4 & 6 & 4 & 5 & 2 \\
\hline & & & $(100)$ & $(83.3)$ & (83.3) & $(66.7)$ & $(66.7)$ & $(66.7)$ & (83.3) & $(66.7)$ & $(100)$ & $(66.7)$ & $(100)$ & $(66.7)$ & $(83.3)$ & $(3:$ \\
\hline & & $\mathrm{R}$ & 0 & 0 & 0 & 2 & 1 & 0 & 1 & 0 & 0 & 0 & 0 & 0 & 0 & 0 \\
\hline & & & (0) & (0) & (0) & $(100)$ & (50) & (0) & (50) & (0) & (0) & (0) & (0) & (0) & (0) & (0) \\
\hline & & I & 0 & 0 & 0 & 0 & 0 & 0 & 1 & 0 & 1 & 0 & 0 & 0 & 0 & 1 \\
\hline & & & (0) & (0) & (0) & (0) & (0) & (0) & (50) & (0) & (50) & (0) & (0) & (0) & (0) & $(5 c$ \\
\hline $\begin{array}{l}\text { Total № of } \\
\text { isolates }\end{array}$ & 19 & $S$ & $\begin{array}{l}14 \\
(73.7)\end{array}$ & $\begin{array}{l}17 \\
(89.5)\end{array}$ & $\begin{array}{l}16 \\
(84.2)\end{array}$ & $\begin{array}{l}14 \\
(73.7)\end{array}$ & $\begin{array}{l}13 \\
(68.4)\end{array}$ & $\begin{array}{l}15 \\
(78.9)\end{array}$ & $\begin{array}{l}11 \\
(57.9)\end{array}$ & $\begin{array}{l}15 \\
(78.9)\end{array}$ & $\begin{array}{l}16 \\
(84.2)\end{array}$ & $\begin{array}{l}17 \\
(89.5)\end{array}$ & $\begin{array}{l}17 \\
(89.5)\end{array}$ & $\begin{array}{l}16 \\
(84.2)\end{array}$ & $\begin{array}{l}14 \\
(73.7)\end{array}$ & $\begin{array}{l}6 \\
(31\end{array}$ \\
\hline & & $\mathrm{R}$ & $\begin{array}{l}4 \\
(21.1)\end{array}$ & $\begin{array}{l}2 \\
(10.5)\end{array}$ & $\begin{array}{l}3 \\
(15.8)\end{array}$ & $\begin{array}{l}4 \\
(21.1)\end{array}$ & $\begin{array}{l}5 \\
(26.3)\end{array}$ & $\begin{array}{l}3 \\
(15.8)\end{array}$ & $\begin{array}{l}7 \\
(36.8)\end{array}$ & $\begin{array}{l}4 \\
(21.1)\end{array}$ & $\begin{array}{l}2 \\
(10.5)\end{array}$ & $\begin{array}{l}2 \\
(10.5)\end{array}$ & $\begin{array}{l}1 \\
(5.3)\end{array}$ & $\begin{array}{l}3 \\
(15.8)\end{array}$ & $\begin{array}{l}4 \\
(21.1)\end{array}$ & $\begin{array}{l}12 \\
(6:\end{array}$ \\
\hline & & I & 1 & 0 & 0 & 1 & 1 & 1 & 1 & 0 & 1 & 0 & 1 & 0 & 1 & 1 \\
\hline & & & (5.3) & (0) & (0) & (5.3) & (5.3) & (5.3) & (5.3) & (0) & (5.3) & (0) & (5.3) & (0) & (5.3) & (5. \\
\hline
\end{tabular}

Table 3: Antibiotic susceptibility pattern of Gram positive isolates in selected health facilities of Addis Ababa, Ethiopia, March-June, 2019.

$\mathrm{AMX}=$ Amoxicillin, $\mathrm{P}=$ Penicillin, $\mathrm{CTX}=$ Cefotaxime, $\mathrm{CRO}=$ Ceftriaxone, $\mathrm{C}=$ Chloramphenicol, $\mathrm{ETP}=$ Ertapenem, $\mathrm{E}=$ Erythromycin,LEV=Levofloxacin, $\mathrm{LNZ}=$ Linezolid, MEM= Meropenem, MXF= Moxifloxacin,OFL= Ofloxacin, TEL= Telithromycin, TE= Tetracycline, SXT= Trimethoprim/Sulfamethoxazole, VA= Vancomycin, $\mathrm{S}=$ Susceptible, $\mathrm{R}=$ Resistant, $\mathrm{I}=$ Intermediate

E. coli were sensitive for cefoxitin (92.9\%), levofloxacin (92.9\%), cefepime (92.9\%), amikacin (92.9\%), piperacillin/ tazobactam (85.7\%), cefazolin (89.3\%), tobramycin (96.4\%), nitrofurantoin (89.3\%), trimethoprim/ sulfamethoxazole (82.1\%), ciprofloxacin (89.3\%), gentamicin (89.3\%), ceftriaxone (82.1\%), and resistant for ampicillin (57.1\%), ceftazidime (39.3\%).

K. Pneumonia isolates showed resistance to ceftazidime (66.7\%), cefepime (50\%), meropenem (50\%), while it was susceptible for cefoxitin (100\%), levofloxacin (100\%), piperacillin/ tazobactam (100\%), cefazolin (83.3\%), amikacin (83.3\%), gentamicin (100\%), tobramycin (100\%), nitrofurantoin (83.3\%), trimethoprim/ sulfamethoxazole (100\%), ciprofloxacin (100\%),

Gram positive bacteria showed variety of susceptibility pattern ranging from $0 \%$ to $100 \%$. The most dominant gram positive bacteria, S. aureus were resistant to trimethoprim/ sulfamethoxazole (50.0\%) and tetracycline (66.7\%) while it showed sensitivity to amoxicillin (100\%), linezolid (100\%), moxifloxacin (100\%), telithromycin (100\%). 
Similarly, S. saprophyticus were susceptible to amoxicillin (100\%), penicillin (100\%), cefotaxime (100\%), ertapenem (100\%), and developed resistance to erythromycin (50\%), chloramphenicol (50\%), ceftriaxone (100\%).

\subsection{Fetal outcome and its association with UTI}

From the total of 424 pregnant women there were 57 (13\%) and 367 (87\%) poor and good fetal outcomes respectively. Poor fetal outcome includes the occurrence of either one or more of the following: APGAR score in 5-min (below 7), low birth weight (below 2500 gm), Premature rupture of membrane, Preterm labor, or IUFD. The presence of bacteriuria, had a significant association with fetal outcomes with ( $p$ value $=0.000$ ). Maternal age, gravidity, educational status, occupation, marital status and history of UTI had no association with the UTI status of the mother (Figure 3).

PROM had a significant association with the occurrence of maternal UTI ( $\mathrm{P}=0.000)$. However, no significant differences were noted between pregnant mothers with or without UTI in terms of IUFD, birth weight, 5 -min Apgar scores and preterm labor, $(20.0 \%$ vs. $80.0 \% ; \mathrm{P}=0.876,20.0 \%$ vs. $80.0 \% ; p=0.521,17.4 \%$ vs. $82.6 \% ; p=0.817$ and $20.0 \%$ vs. $80.0 \% ; p=0.846$ ) respectively. (Table 4 )

Table 4: Association of significant bacteriuria with IUFD, PROM, birth weight, APGAR score and GA at birth

\begin{tabular}{|c|c|c|c|c|c|c|}
\hline \multirow[t]{3}{*}{ Variables } & & \multirow{2}{*}{\multicolumn{2}{|c|}{$\begin{array}{l}\text { Bacteriuria } \\
\text { № (\%) }\end{array}$}} & \multirow[t]{3}{*}{$\operatorname{COR}(95 \% \mathrm{Cl})$} & \multirow[t]{3}{*}{ AOR $(95 \% \mathrm{Cl})$} & \multirow[t]{3}{*}{$P$ value } \\
\hline & & & & & & \\
\hline & & No & Yes & & & \\
\hline \multirow[t]{2}{*}{ IUFD } & No & $357(85.2)$ & $62(14.8)$ & 1.4 & 1.2 & 0.876 \\
\hline & Yes & $4(80.0)$ & $1(20.0)$ & $(0.16,3.09)$ & $(0.13,11.29)$ & \\
\hline \multirow[t]{2}{*}{ PROM } & No & $349(87.7)$ & $49(12.3)$ & 8.3 & 8.4 & $0.000 *$ \\
\hline & Yes & $12(46.2)$ & $14(53.8)$ & $(3.63,18.99)$ & $(3.60,19.41)$ & \\
\hline \multirow[t]{2}{*}{ Birth weight } & Normal & $345(85.4)$ & $59(14.6)$ & 1.5 & 1.5 & 0.521 \\
\hline & Under & $16(80.0)$ & $4(20.0)$ & $(0.47,4.53)$ & $(0.47,4.50)$ & \\
\hline \multirow[t]{2}{*}{ APGAR score } & Normal & $342(85.3)$ & $59(14.7)$ & 1.2 & 1.1 & 0.817 \\
\hline & Low & $19(82.6)$ & $4(17.4)$ & $(0.40,3.71)$ & $(0.37,3.50)$ & \\
\hline \multirow[t]{2}{*}{ Gestational age at birth } & Term & $357(85.2)$ & $62(14.8)$ & 1.4 & 1.2 & 0.846 \\
\hline & Preterm & $4(80.0)$ & $1(20.0)$ & $(0.16,13.09)$ & $(0.14,11.55$ & \\
\hline
\end{tabular}

As indicated in table 5 below, out of the 63 pregnant mothers with bacteriuria, 18 (28.6\%) of them gave birth with poor fetal outcome. The mothers with positive bacteriuria were found significantly associated with having a poor fetal outcome which is a summative effect of individual adverse fetal outcomes $(95 \% \mathrm{Cl}=1.742,6.261)$.

Table 5: Association of significant bacteriuria with fetal outcome, keeping other variables constant

\begin{tabular}{|llllll|}
\hline Variables & Good fetal outcome & Poor fetal outcome & COR (95\% Cl) & AOR (95\% Cl) & P value(95\% Cl) \\
\hline UTI & $322(89.2 \%)$ & $39(10.8 \%)$ & 1 & 1 & $0.000^{*}$ \\
Negative & $45(71.4 \%)$ & $18(28.6 \%)$ & 3.3 & 3.2 & $(1.742,6.261)$ \\
Positive & & & & \\
\hline Educational level & $61(16.6 \%)$ & $7(12.3 \%)$ & 1 & 1 & 0.223 \\
No formal education & $148(40.3 \%)$ & $25(43.9 \%)$ & 0.5 & 0.6 & $(0.900,1.568)$ \\
Primary school & $125(34.1 \%)$ & $17(29.8 \%)$ & 0.7 & 0.8 & \\
Secondary school & $33(9.0 \%)$ & $8(14.9 \%)$ & 0.6 & 0.6 & \\
Higher education & & & & 1 & 0.058 \\
Gravidity & $235(89.0 \%)$ & $29(11.0 \%)$ & 1 & 1.6 & $(0.981,3.013)$ \\
Multigravida & $132(82.5 \%)$ & $28(17.5 \%)$ & 1.7 & & \\
Primigravida & & & & & \\
\hline
\end{tabular}

\section{Discussion}


The study was done to determine the magnitude and drug resistance profile of bacterial species isolated from pregnant women during the time of their third trimester and to check the association between UTI and adverse fetal outcomes. The overall prevalence of significant bacteriuria in this study was $14.9 \%$ (63/424), the result correlates with the finding of similar study conducted in Dire Dawa (14\%), Harar (15.5\%), Gondar (10.4\%) but lower than the studies conducted in Nigeria (85\%), Benin (74\%) and Goba and Sinana Woredas, Bale Zone, South-East Ethiopia, (35.3\%) [6, 12, 27-30]. Sample size, trimester of pregnant women and demographic difference might be mentioned as the possible reason for the variation.

The prevalence of UTI among symptomatic pregnant women in this study was lower than the prevalence of asymptomatic at a rate of $3.3 \%$ and $11.6 \%$ respectively. The prevalence of symptomatic bacteriuria in the study site was in agreement with the result from similar studies done in Gondar (3.4\%), lower than the result from Harar (21\%), Dire Dawa (8\%), Yemen (66.6\%) but slightly higher than the result from Bahir Dar $(1.9 \%)[6,8,12,17,27]$.

With respect to asymptomatic bacteriuria, the finding from Harar, Ethiopia, (13.0\%), matches with this study but lower incidence rate was reported in Bahir Dar, Ethiopia, (7.6\%), Dire Dawa, Ethiopia, (5.9\%), and Gondar, Ethiopia, (7.0\%) [6, 12, 17]. Higher prevalence of asymptomatic and symptomatic UTI reported in Yemen might be attributed to the less sample size, geographical variation or may also be due to difficulties in interpreting the symptoms by the study participants or the data collectors.

Sixty eight percent (68.75\%) of the total bacterial isolates belongs to gram negative similar to the study conducted in Gondar (67.5), Dire Dawa (73.1), Yemen (63.4\%), Tanzaniya (61.9\%), but lower than the report from Harar (90.3\%), and India (100\%)[6, 8, 12, 27, 31, 32]. In addition to the sample size variation, the environmental conditions, population difference and socioeconomic status of the participants can be mentioned as possible reason for the result variation.

With regard to bacterial isolates, $E$. coli was the most dominant bacterial isolate (44.4\% and 63.6\%) from the total and from gram negative isolates respectively. Similarly, high proportions of E. coli from UTI positive pregnant women were reported in Nigeria (66.6\%), India (43.9\%), Dire Dawa (34.6\%), Gondar (47.5\%), and Tanzania (33.3\%) [2, 6, 12,31,33]. In most of these cases, either Kleibssiela spp or coagulase negative Staphylococcus are the second predominant organisms next to $E$. coli. In contrast to the above findings, Citrobacter freundii were reported by Tula and Lyoha in Nigeria as the most dominant bacterial isolate from UTI positive samples [34].

In this study, K Pneumoniea and $S$. aureus were equally ranked as the second predominant pathogens while $S$. epidermidis and $E$. faecalis ranked third. These findings indicate that even though there are a number of UTI causing bacterial species, majority of UTI cases are limited to few groups of bacteria.

Amikacin, ciprofloxacin, gentamicin, cefoxitin, tobramycin and levofloxacin were the most effective drugs over gram negative bacteria by producing more than $90 \%$ susceptibility.

In the study conducted by Alemu et al., ceftriaxone, ciprofloxacin, norfloxacin, gentamicin, amoxicillin-clavulanic acid, co-trimoxazole and tetracycline produced the highest level of sensitivity over gram negative bacteria [6]. Disk diffusion and MIC breakpoints for norfloxacin are deleted from CLSI M100, 29th ed. [35]. Similarly, ceftriaxone, gentamicin and ciprofloxacin were the most effective drugs against gram negative bacteria in the study done by Derese [12].

In Libya, nitrofurantoin, amikacin, ofloxacin, ciprofloxacin and cefotaxime were reported as drugs that produced high susceptibility to most of the isolates [15].

From gram negative bacteria, E. coli showed sensitivity to all the antibiotics tested. On contrary, a study done in Mekelle, Northern Ethiopia, reported a resistance of E. coli to amoxicillin-clavulnic acid and trimethoprim / sulfamethoxazole and ampicillin [36].

Similarly S. aureus also showed resistance to tetracycline but was sensitive to most of the antibiotics tested, this result differs from the result reported in Mekelle [36].

When we compare the rate of isolates of Gram negative and Gram positive bacteria. Gram negative bacteria were the dominant causative agent of UTI which is in line with others report $[12,15,36]$.

In this study, the association of low birth weight and maternal UTI were not significant $(P=0.521)$. About $16(80 \%)$ and $4(20 \%)$ underweight deliveries were recorded from 361 UTI negative and 63 UTI positive pregnant mothers respectively. This proportion of low birth weight were in line with the study conducted by Iqbal et al., However; in a study conducted by Amiri and his colleague in Ghana, birth weight were significantly associated with maternal UTI status [18, 19, 37]. The difference in sample size, difference in settings and sampling method can explain the difference in the association of the two variables in different geography.

Like birth weight, APGAR score (in the 1st and 5th minute), IUFD and gestational age were not statistically associated with maternal UTI; however, in a study conducted by Siakwa in Ghana, occurrence of low APGAR score (less than 7) and preterm labor respectively were 3.8 and 3.4 times than their occurrence in UTI negative mothers. A recent study done by Farah Iqbal also reported similar association of preterm delivery and low birth weight with UTI. A retrospective and observational study in Brazil further points out the significant association that exist between preterm birth and UTI but with respect to APGAR score, the result had similar finding with this study. The difference in significance level and odds of occurrence may be attributed to the socio-demographic difference of study subjects. $[8,19,38]$.

The finding of this study indicates that UTI during pregnancy had significant association with the occurrence of $P R O M(P=0.000, A O R=8.4, C l=3.60,19.41)$. This finding agrees with the finding of Siakwa M., et al. in Ghana, Antonio E. in Brazil. [19, 38].

According to different studies, UTI induces macrophages to release metalloproteinase enzymes that can degrade amniotic membranes and ultimately leads to rupture of amniotic membranes. [19,38]. 
Our findings have some limitations, like the sample size though enough to describe the magnitude of UTI, it looks small while we are considering the fetal outcome. Second, being a cross sectional study, the exact roles of UTI in pregnant women and fetal outcome is confounded. If a follow up study were followed, we could understand the role of UTI in fetal outcomes during the course of pregnancy.

\section{Conclusion}

The finding from this study showed that the prevalence of UTI in the study area was high in general and higher proportion was taken by pregnant women without symptoms. Gram negative bacteria remained as the dominant causative agents of UTI. E. coli, S. aureus and K. Pneumoniae were the frequent isolates. The antibiotic susceptibility test showed variety of drug resistance pattern among different bacterial isolates. E. coli were susceptible for most of the antibiotics tested. Majority of gram positive isolates were resistant for Tetracycline and the two antibiotics: Tetracycline and Trimethoprim/Sulfamethoxazole were not effective against the most prevalent gram positive bacteria, $S$. aureus. UTI was found significantly associated with fetal outcome after keeping all other variables constant. Early screening of UTI in pregnant women is useful to minimize complication related to fetal outcomes. The exact roles of UTI in fetal outcomes during pregnancy needs further research works with better study design and sample size.

\section{Abbreviations}

ANC Antenatal Care

ANC Antenatal care clinic

APGAR Activity Pulse Grimace Appearance Respiration

ART Antiretroviral therapy

AST Antimicrobial Susceptibility Tests

AYH Adolescence and youth health

BAP Blood Agar Plate

CFU Colony forming unit

CoNS Coagulase- Negative Staphylococcus

EPHI Ethiopian Public Health Institute

IUGR Intrauterine Growth Retardation

MAC MacConkey Agar Plate

NRLCBM National referral laboratory clinical bacteriology and mycology

PROM Pre Rupture of Membrane

SOP Standard Operating Procedure

UTI Urinary Tract Infection

\section{Declarations}

\section{Ethics approval and consent to participate}

The ethical approval for this study was obtained from Department Research and Ethical Review Committee (DRERC) of Department of Medical Laboratory Sciences, College of Health Sciences, Addis Ababa University, and Addis Ababa Health Bureau prior to initiation of the study. Support letters were written from the department to Addis Ababa Health Bureau, EPHI, Gerji Health Center, Felege Meles Health Center and Tikur Anbessa Speclised Hospital.

\section{Informed consent was obtained from all participants}

Proper explanation about the study was given to the study participants and the study was conducted after obtaining full verbal and written consent from pregnant women attending at antenatal clinics at the study sites.

The purpose of the study was explained to the study participants and also they were informed that the procedures used in the study do not cause any harm to them or their child. They were told that they have a full right to participate or not, to withdraw the consent and stop participation at any time without any form of prejudice.

Study participants were also informed that confidentiality of the information and privacy of the respondents will be assured by using codes instead of their names at each step of the study process. In addition, they were informed about the benefit of being part of the study. Positive UTI results were given to clinician and the study participants got appropriate treatment. All methods were performed in accordance with the relevant guidelines and regulations. 
Consent for publication: Not applicable

\section{Availability of data and materials}

Declaration: The datasets used and/or analysed during the current study are available from the corresponding author on reasonable request.

Competing interests: The authors declare that they have no competing interests.

Funding: Ethiopian Public Health Institute, NRLCBM-National referral laboratory clinical bacteriology and mycology have funded the culture media and laboratory setup for this research.

Authors' contributions: WZ, KD, DD: Conceive the study protocols. WZ, AA, YY: Done the laboratory procedures. DD, HY: Done follow up of the fetal outcomes. WZ: Data analysis. WZ, KD: interpretation of Data. All authors reviewed the manuscript.

Acknowledgements: We would like to express deepest gratitude to our study participants, data collectors, all Antenatal Care (ANC) staff members of Tikur Anbessa Specialized Hospital, Gerji Health Center, and Felege Meles Health center.

Our special thanks goes to Mr. Dawit Assefa (quality officer, NRLCBM) and Mr. Estifanos Tsigie (NRLCBM laboratory team leader), Mr. Tesfaye Legesse (Food microbiology) and Mrs. Mulushewa Gebreegziabher (Media preparation room, NRLCBM laboratory) for their unreserved support.

We want to acknowledge Addisalem Zebene, for her unreserved support and comments during the study period.

\section{References}

1. Annaldasula AR. A Study on Urinary Tract Infections in Pregnancy. Ann.Int. Med. Den. Res. 2018; 4(2)

2. Joachim O., Gideon I., Ruth A., Onyibo S. Isolation, Identification and Antibiotic Susceptibility Pattern of Bacteria Associated with Urinary Tract Infections (UTIs) in Pregnant Women Attending Antenatal care at Regina-Caeli Hospital and Maternity Awka, Anambra State, Nigeria. World Journal of Pharmaceutical Research 2016. 5,( Issue 3): 215-226.

3. Pradhan, B. and Pradhan SB., Prevalence of Urinary Tract Infection and Antibiotic Susceptibility Pattern to Urinary Pathogens in Kathmandu Medical College and Teaching Hospital, Duwakot. Birat Journal of Health Sciences, 2017. 2(1): 134-137.

4. Alsamarai M., Khorshed A., Ali H. Urinary tract infection in female in Kirkuk city, Iraq: Association between risk factors and bacterial type. Our Dermatology Online, 2017. 8(3): 242.

5. Iqbal, F., Zehra K., Ashfaq S., Memon E., Aziz S. Sultan S., et al., Association of Maternal Lower Urinary Tract Infection with Adverse Fetal Outcome in Terms of Preterm Labor and Low Birth Weight J. Soc. Obstet. Gynaecol., 2017. 7(1):33-37.

6. Alemu, A., Moges F., Shiferaw Y., Tafess K., Kassu A., Anagaw B., et al. Bacterial profile and drug susceptibility pattern of urinary tract infection in pregnant women at University of Gondar Teaching Hospital, Northwest Ethiopia. BMC research notes, 2012. 5(1): 197.

7. Puca, E., Urinary Tract Infection in Adults. Clinical Microbiology: Open Access, 2014. 3(6).

8. AL-Kadassy, A., Baraheem O., Ogaili M, The Prevalence of Urinary Tract Infection (UTI) Among Pregnant Women In Hodeidah City, Yemen. IOSR-J Dent Med Sci, 2016. 15(12): 105-9.

9. Lavrinenko, A., Tishkambayev Y., Serbo Y., Shambilova N., Azizov I., Sandle T., et al. Prevalence Of Urinary Tract Infections On Pregnant Women. Archives of the Balkan Medical Union, 2016. 51(3).

10. Schnarr, J., Smaill F, Asymptomatic bacteriuria and symptomatic urinary tract infections in pregnancy. European journal of clinical investigation, 2008. 38 : 50-57.

11. Szweda, H., Jóźwik M., Urinary tract infections during pregnancy-an updated overview. Dev. Period Med, 2016. 20: $263-272$.

12. Derese, B., Kedir H., Teklemariam Z., Weldegebreal F., Balakrishnan S., Bacterial profile of urinary tract infection and antimicrobial susceptibility pattern among pregnant women attending at antenatal Clinic in Dil Chora Referral Hospital, Dire Dawa, Eastern Ethiopia. Therapeutics and clinical risk management, 2016. 12: 251.

13. Zeyaullah M., Kaul V. Prevalence of urinary tract infection and antibiotic resistance pattern in Saudi Arabia population. Global Journal of Biology, Agriculture \& Health Sciences, 2015. 4(1): 206-214.

14. Getu Y., Prevalence and Drug Susceptibility Pattern Of Bacteria Associated With Urinary Tract Infection Among HIV Positive Patients Attending Alert Center, Addis Abeba Ethiopia in Department of Medical Laboratory Sciences. 2015, Addis Ababa University: Addis Ababa. Ethiopia.

15. Tamalli, M., S. Bioprabhu, M. Alghazal, Urinary tract infection during pregnancy at Al-khoms, Libya. International Journal of Medicine and Medical Sciences, 2013. 3(5): 455-459.

16. Stanley Chukwudozie Onuoha, Kayode Fatokun. Prevalence and antimicrobial susceptibility pattern of Urinary Tract Infection (UTI) among pregnant women in Afikpo, Ebonyi State, Nigeria American Journal of Life Sciences 2014. 2(2): 46-52.

17. Demilie, T., Beyene G., Melaku S., Tsegaye W., Urinary bacterial profile and antibiotic susceptibility pattern among pregnant women in North West Ethiopia. Ethiopian journal of health sciences, 2012. 22(2).

18. Amiri, M., Lavasani Z., Norouzirad R., Najibpour R., Mohamadpour M. Nikpoor R., et al., Prevalence of urinary tract infection among pregnant women and its complications in their newborns during the birth in the hospitals of Dezful city, Iran, 2012-2013. Iranian Red Crescent Medical Journal, 2015. 17(8). 
19. Siakwa M, Kpikpitse D., Azanu W, John E., Doe F., Ebu I., et al. Maternal And Perinatal Outcomes Among Pregnant Women With Urinary Tract Infection. International Journal of Current Research 2016. 8(06): 33366-33371.

20. Alsamarai, A. and Ali S, Urinary tract infection in Female in Kirkuk city, Iraq: Causative agents and antibiogram. WJPPS, 2016. 5:261-273.

21. Merga, Y., Terfa K., Mamuye D., Bitew A., Antimicrobial Susceptibility Profile of Bacterial Uropathogens Isolated from Pediatric Patients at Yekatit 12 Hospital Medical College in Department of Medical Laboratory Sciences 2014, Addis Ababa University Addis Ababa.

22. Eshwarappa M, Dosegowda R, Aprameya IV, Khan MW, Kumar PS, Kempegowda P. Clinico-microbiological profile of urinary tract infection in south India. Indian J Nephrol. 2011;21(1):30-36.

23. Prakash, D. and R.S. Saxena, Distribution and antimicrobial susceptibility pattern of bacterial pathogens causing urinary tract infection in urban community of meerut city, India. ISRN microbiology, 2013.

24. Wasnik, D.D. and Tumane PM, Prevalence and antibacterial susceptibility pattern of urinary tract infection causing human pathogenic bacteria. Asian Journal of Biomedical and Pharmaceutical Sciences, 2012. 2(15).

25. Perera, J., Cyril R., Piyumi P., Nimesha G., Renuka J., Asymptomatic bacteriuria in pregnancy: prevalence, risk factors and causative organisms. Sri Lankan Journal of Infectious Diseases, 2012. 2(1).

26. Lega, T., "Bacterial Uropathogens and their Drug Resistance Pattern in Diabetic Patients Attending Yekatit 12 Hospital Medical College, Addis Ababa, Ethiopia”. 2015, Addis Ababa University: Addis Ababa.

27. Workneh, A., Antimicrobial susceptibility Pattern of Bacterial Uropathogens and Associated Factors of Urinary Tract Infection Among Pregnant Women Attending Antenatal Care Clinic of Hiwot Fana Specialized University Hospital, Harar, Eastern Ethiopia. 2018, Addis Ababa University.

28. Dougnon TV, Bankole H., Fanou B., Ahoyo A., Hounmanou G., Gbaguidi C., et. al., Burden of Urinary Tract Infections Among Pregnant Women Attending the Departmental Hospital of Ouémé-Plateau in Porto-Novo, Benin. 2015 September;05(09) Suppl 02: S1-S8. J P h a r m B i o m e d S c i 2015. 05(09).

29. Turay, A., Eke S., Oleghe P., Ozekhome M., The prevalence of urinary tract infections among pregnant women attending antenatal clinic at Ujoelen primary health care centre, Ekpoma, Edo state, Nigeria. International Journal of Basic, Applied and Innovative Research, 2014. 3(3): 86-94.

30. Taye, S.,Getachew M., Desalegn Z., Biratu A., Mubashir K. Bacterial profile, antibiotic susceptibility pattern and associated factors among pregnant women with Urinary Tract Infection in Goba and Sinana Woredas, Bale Zone, Southeast Ethiopia. BMC research notes, 2018. 11(1): 799.

31. Moyo, S.J., Aboud S., Kasubi M., Maselle Y. Bacterial isolates and drug susceptibility patterns of urinary tract infection among pregnant women at Muhimbili National Hospital in Tanzania. Tanzania Journal of Health Research, 2010. 12(4): 233-236.

32. Ranjan, A., Sridhar K., Matta N., Chokkakula S., Ansari K. Prevalence of UTI among Pregnant Women and Its Complications in Newborns. Indian Journal of Pharmacy Practice, 2017. 10(1): 45.

33. Pati K., R.K.L., Lenka K., Sahu C. Antibiotic resistance pattern in pregnant women with urinary tract infections in a tertiary care Indian teaching hospital. International Journal of Applied Research, 2017. 3(12): 231-235.

34. Tula M. and lyoha O, Distribution and antibiotic susceptibility pattern of bacterial pathogens causing urinary tract infection in Mubi General Hospital, YolaNigeria. British Journal of Medicine and Medical Research, 2014. 4(19): 3591.

35. CLSI.Performance standards for antimicrobial susceptibility testing. $29^{\text {th }}$ ed. CLSI supplement M100. Wayne.PA: Clinical and Laboratory Standards Institute; 2019.

36. Tsegay, E., Bacterial Profile And Drug Susceptibility Pattern Of Urinary Tract Infection In Pregnant Women Attending Antenatal Care At Mekelle Hospital, Mekelle, Northern Ethiopia.Department Of Microbiology, Immunology and Parasitology 2014, Addis Ababa Universit: Addis Ababa.

37. Iqbal F. Naqvi Z., Ashfaq S., Memom E., Aziz S., Sultan S. et al., Factors associated with infants' and young children's (6-23 months) dietary diversity in Pakistan: evidence from the demographic and health survey 2012-13. Nutrition journal, 2017. 16(1): 78.

38. Antonio E. Urinary Tract Infection in high risk pregnant women. Revista De Pathologia Tropical, 2013. 42 (1): 21-29

\section{Figures}




\section{Magnitude of UTI}

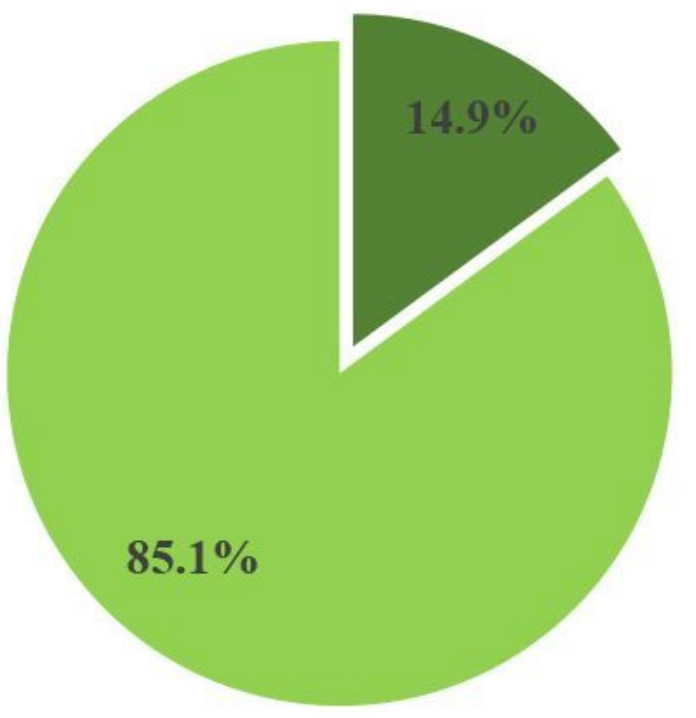

- UTI postive

- UTI negative

\section{Figure 1}

Magnitude of UTI in selected health facilities of Addis Ababa, Ethiopia, March-June, 2019.

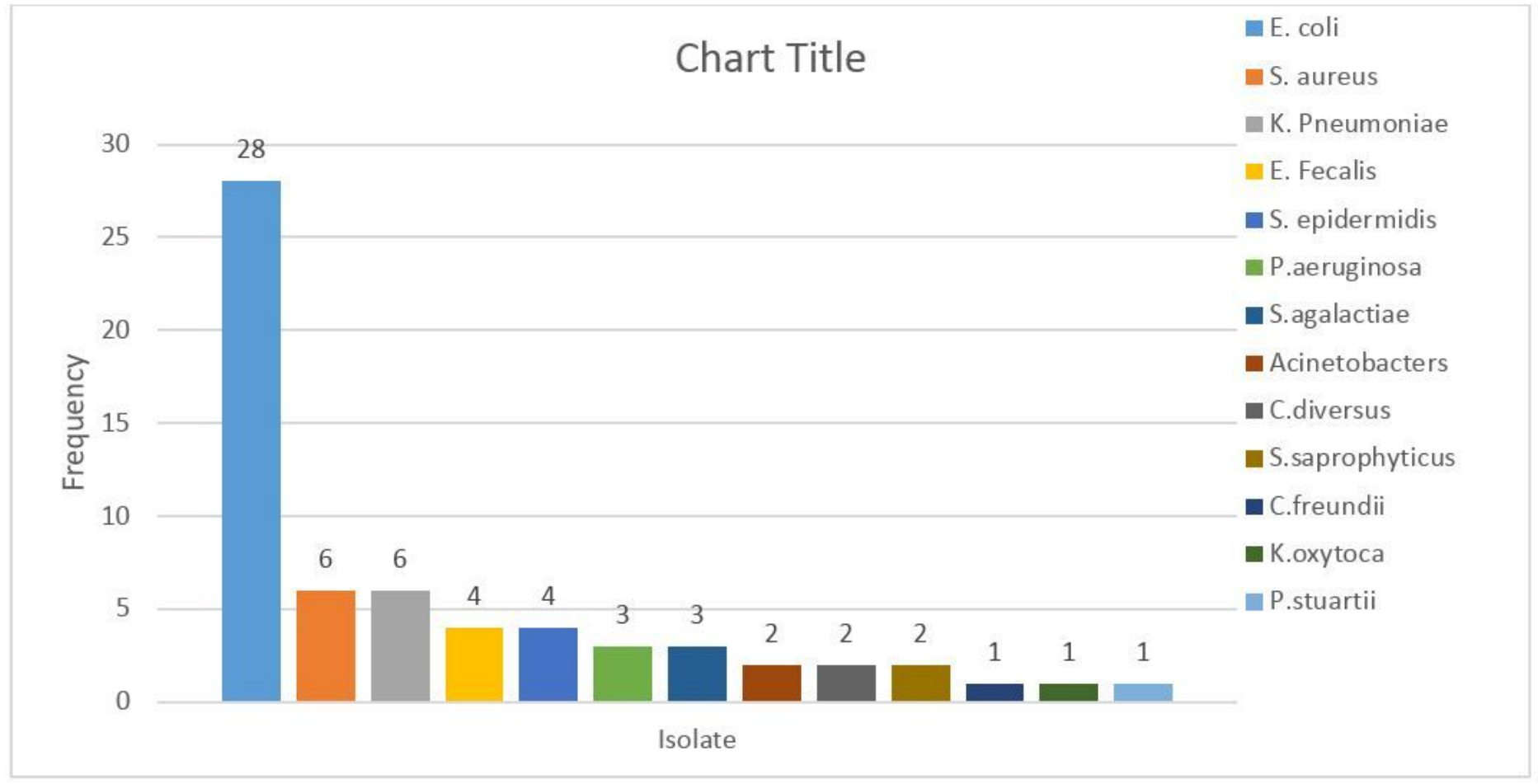

\section{Figure 2}

Distribution of bacterial species isolated from pregnant women, in selected health facilities of Addis Ababa, Ethiopia, March-June, 2019. 


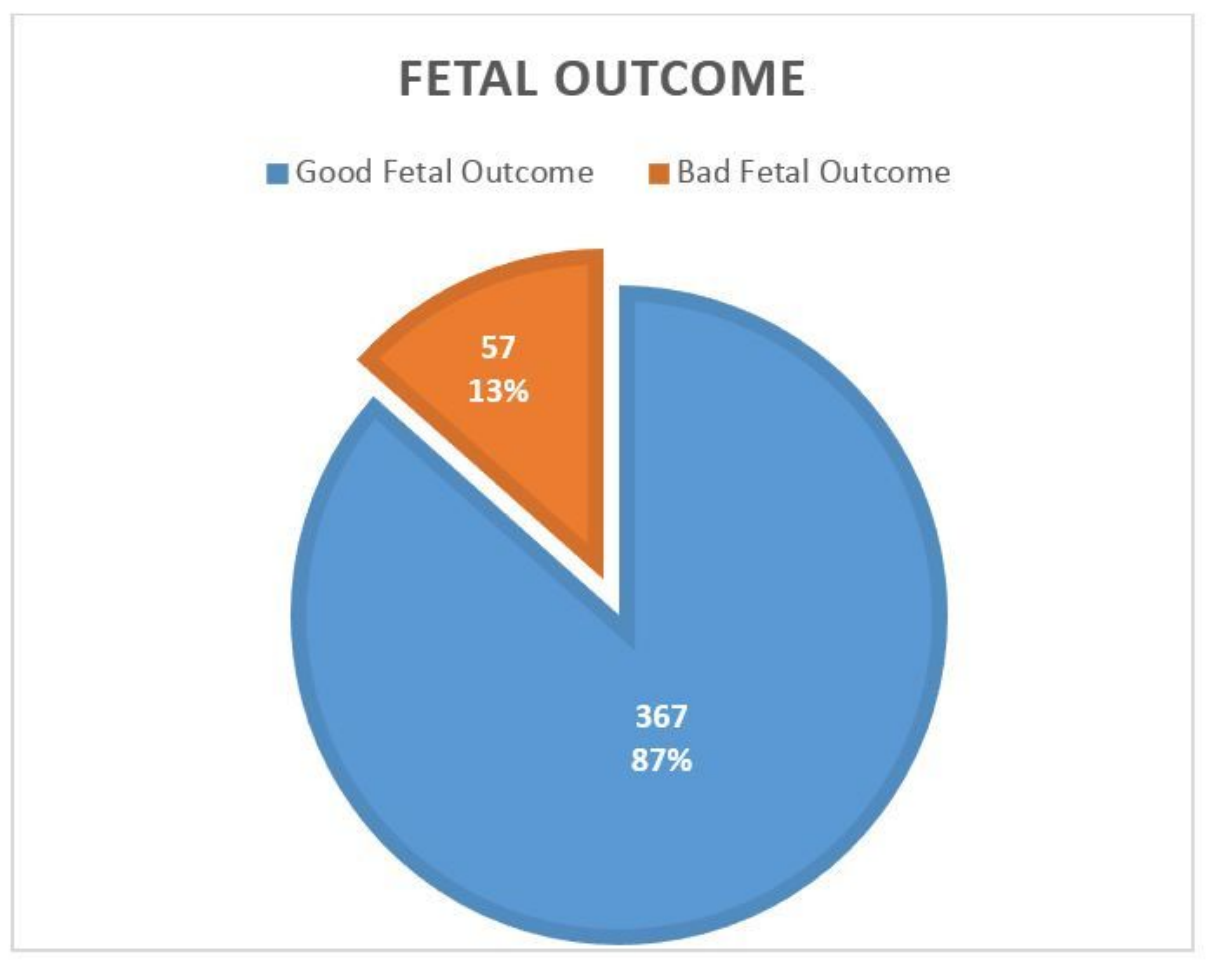

Figure 3

Fetal outcome of pregnant women, 2019. 